\title{
Novel Criterion for Synchronization Stability of Complex Dynamical Networks with Non-delayed and Delayed Coupling
}

\author{
Detian Huang, Jianeng Tang ${ }^{*}$, Peizhong Liu and Yuzhao Zhang \\ College of Engineering, Huaqiao University, No.269, Chenghua North Road, \\ Fengze District, Quanzhou, Fujian 362021, PR China \\ *Corresponding author's email:tangjianeng@sina.com
}

\begin{abstract}
In the paper, a class of complex dynamical network with non-delayed and delayed coupling is investigated. Then the problem of synchronization stability analysis for the complex dynamical network is further discussed. Taking advantage of linear matrix inequalities (LMI), a novel Lyapunov function is constructed. And then a new general stability criterion is proposed of synchronization state in the complex dynamical networks. Finally, numerical simulations are provided to show the effectiveness and feasibility of the proposed theorem.
\end{abstract}

Keywords: synchronization stability; complex networks; linear matrix inequalities; Lyapunov function

\section{Introduction}

Complex networks model has attracted great attention in the past decades [1-5]. One of the most important reasons is that most practical systems can be modeled by complex dynamical networks. The synchronization phenomenon is one of the most important dynamical properties of complex networks. And many real world complex networks usually display better cooperative or synchronous behaviors, such as the synchronous motion on the internet, synchronous transfer of digital or analog signals in communication networks, and biological neural networks relating with synchronization. There are a lot of literatures about synchronization stability analysis for complex networks [6-10].

In practice, complex networks with delay coupling are considered because of the network traffic congestions as well as the finite speed of signal transmission. The synchronization for all kinds of networks with delay coupling has attracted much attention from researchers in different fields. Recently, $\mathrm{Li}$ and Chen introduced the uniform dynamical network model to include coupling delays among the network nodes and studied its synchronization [11]. Lü and Chen [12] studied the synchronization of time-varying complex dynamical networks in which the inner-couplings are time-varying. Gao et al. $[13,14]$ presented several new delay-dependent conditions for a general complex network model with coupling delay. Then synchronization in complex networks with delay coupling was researched extensively and deeply, and some synchronization criteria were derived [15-20]. The synchronization stability problem of general complex dynamical networks with non-delayed and delayed coupling is investigated based on a piecewise analysis method [21]. It is worth noting that little progress has been made toward complex dynamical networks with non-delayed and delayed coupling. Therefore, as one of the fundamental problems for complex networks, synchronization stability analysis for complex dynamical networks still remains unsolved and challenging. Based on the above analysis, it is our purpose to propose novel delay-dependent conditions for continuoustime networks. 
In the paper, the synchronization stability problem of a general complex dynamical networks model with non-delayed and delayed coupling is further discussed. Taking advantage of linear matrix inequalities (LMI), a novel Lyapunov function is constructed. And then a new general stability criterion is proposed about synchronization state in the complex dynamical networks. The rest of this paper is organized as follows. We review a classical complex dynamical network model and some relevant results in the literature on synchronization in Section 2. A new delay-dependent synchronization stability conditions for continuous-time networks is presented in Section 3. In Section 4, some illustrative examples are provided to show the effectiveness, and the conclusion is presented in Section 5.

Notation: The notation used throughout the paper is fairly standard. Let $R^{n}$ denotes the $n$-dimensional Euclidean space over the reals with the norm $\|\cdot\|$. For any $u=\left(u_{i}\right)_{1 \leq i \leq n}$, $v=\left(v_{i}\right)_{1 \leq i \leq n}$ and $u, v \in R^{n}$, we define the scalar product of the vectors $u$ and $v$ as: $\langle u, v\rangle=\sum_{i=1}^{n} u_{i} v_{i} \quad$. Let $R=(-\infty,+\infty) \quad, \quad R_{+}=[0,+\infty) \quad, \quad R_{+}^{*}=(0,+\infty) \quad$, $R_{+}^{* n}=\left\{v=\left(v_{i}\right)_{1 \leq i \leq n} \in R^{n}, v_{i} \in R_{+}, \forall i=1,2, \ldots, n\right\}$. Let $\lambda(M)$ denotes the set of eigenvalues of the matrix $M, M^{\prime}$ its transpose and $M^{-1}$ its inverse. We define $|M|=\left(\left|m_{i j}\right|\right)_{1 \leq i, j \leq n}$ if $M=\left(m_{i j}\right)_{1 \leq i, j \leq n}$. Let $C_{n}=C\left([-\tau, 0], R^{n}\right)$ be the Banach space of continuous functions mapping the interval with the topology of uniform convergence. For a given $\phi \in C_{n}$, we define $\|\phi\|=\sup _{-\tau \leq \theta \leq 0}\|\phi(\theta)\|, \phi(\theta) \in R^{n}$. We define the function $\operatorname{sgn}(\cdot)$ and $M^{*}=\left(m_{i j}{ }^{*}\right)_{1 \leq i, j \leq n}$ are

$$
\operatorname{sgn}(\vartheta)=\left\{\begin{array}{l}
1, \quad \vartheta \in R_{+}^{*} \\
-1, \quad-\vartheta \in R_{+}^{*} \\
0, \quad \vartheta=0
\end{array}, \quad m_{i j}^{*}=\left\{\begin{array}{l}
m_{i j}, i=j \\
\left|m_{i j}\right|, i \neq j
\end{array}\right.\right.
$$

\section{Complex Dynamical Networks Model}

In what follows, we consider a general complex dynamical network consisting of $N$ identical linearly and diffusively coupled nodes, with each node being an $n$-dimensional dynamical system and introduce a complex delayed dynamical network model. This dynamical network is described by

$$
\dot{x}_{i}=f\left(x_{i}(t), x_{i}(t-\tau)\right)+c_{1} \sum_{j=1}^{N} C_{i j} G_{1} x_{j}(t)+c_{2} \sum_{j=1}^{N} C_{i j} G_{2} x_{j}(t), i=1,2, \ldots, N
$$

where $x_{i}=\left(x_{i 1}, x_{i 2}, \ldots, x_{i n}\right)^{T} \in R^{n}$ is a state vector representing the state variables of node $i, f(\cdot) \in R^{n}$ is a continuously differentiable vector function, $G_{l}=\left(G_{l i j}\right)_{n \times n} \in R^{n \times n}$ is a constant inner-coupling matrix between node $i$ and node $j(i \neq j)$ for all $1 \leq i, j \leq N$, the constant $c_{i}>0(i=1,2)$ is the coupling strength, time delay $\tau$ is a positive real number, $C=\left(C_{i j}\right)_{N \times N}$ is the coupling configuration matrix representing topological structure of the network, in which $C_{i j}$ s defined as follows: if there is a connection from node $i$ to node $j(i \neq j)$, then $C_{i j}=C_{j i}=1$, otherwise $C_{i j}=C_{j i}=0$, and the diagonal elements of matrix $C$ are defined by

$$
C_{i i}=-\sum_{j=1, j \neq i}^{N} C_{i j}, i=1,2, \ldots, N
$$


Network (1) is a general complex network model in which there exist both non-delayed coupling and delayed coupling. It means that each node communicates with other nodes the information at time $t$ as well as at time $t-\tau$. In fact, this phenomenon exists commonly in our real world. For example, in the stock market, decision-making of single trader is influenced by that of others at time $t$ as well as at time $t-\tau$.

There have been various definitions of synchronization in the literature [4, 21]. Hereafter, the delayed dynamical network (1) is said to achieve (asymptotical) synchronization if

$$
x_{1}(t)=x_{2}(t)=\ldots=x_{N}(t)=s(t), \text { as } t \rightarrow \infty
$$

where $s(t) \in R^{n}$ is a solution of an isolate node, namely, $\dot{s}(t)=f(s(t), s(t-\tau))$.

To obtain the main results, we will need the following lemmas.

Lemma 1. Consider the delayed dynamical network (1). Let

$$
0=\lambda_{1} \leq \lambda_{2} \leq \ldots \lambda_{N}
$$

be the eigenvalues of the outer-coupling matrix $C$. If the following $N-1$ of $n$ dimensional delayed differential equations are asymptotically stable about their zero solutions:

$$
\dot{w}=\left(J_{1}+c_{1} \lambda_{i} G_{1}\right) w(t)+\left(J_{2}+c_{2} \lambda_{i} G_{2}\right) w(t-\tau), i=2, \ldots, N
$$

where $J_{1}$ is the Jacobian of $f\left(x_{i}(t), x_{i}(t-\tau)\right)$ at $s(t)$ and $J_{2}$ is the Jacobian of $f\left(x_{i}(t), x_{i}(t-\tau)\right)$ at $s(t-\tau)$, then the synchronized states (3) are asymptotically stable for the dynamical network (1)[21].

\section{Main Results}

In this section, we will derive the main results about the stability analysis of synchronization for the complex delayed dynamical network (1). Based on the abovementioned assumptions and definitions, we can obtain the following theorem.

Theorem 1. For the dynamical system (5), if there exists $Y=\left(y_{i j}\right)_{1 \leq i, j \leq n}=-A-\tau B$ such that

(1) $y_{i i}>0, i=1,2, \ldots, n$ and $y_{i j} \leq 0$, for $i \neq j, i, j=1,2, \ldots, n$

(2) Successive principal minors of $Y$ are positive, that is,

$$
\operatorname{det}\left(\begin{array}{cccc}
y_{11} & y_{12} & \ldots & y_{1 i} \\
\ldots & \ldots & \ldots & \ldots \\
y_{i 1} & y_{i 2} & \ldots & y_{i i}
\end{array}\right)>0, i=1,2, \ldots, n
$$

where $A=\left(H+c_{2} \lambda_{i} \Gamma\right)^{*} \quad, \quad B=\left|c_{2} \lambda_{i}\right||\Gamma H|+\left(c_{2} \lambda_{i}\right)^{2}\left|\Gamma^{2}\right|, \quad H=J_{1}+c_{1} \lambda_{i} G_{1}$ and $\Gamma=J_{2} /\left(c_{2} \lambda_{i}\right)+G_{2}$, then zero solution of system (5) is asymptotically stable. (1).

That is, the synchronized states (3) are asymptotically stable for the dynamical network

Proof. Suppose that $w(t)$ is continuously differentiable when $t \geq 0$, by using the Newton- Leibniz formula, we can get

$$
w(t-\tau)=w(t)-\int_{t-\tau}^{t} \dot{w}(s) d s
$$

Substituting Eq. (5) into Eq. (6), we achieve

$$
w(t-\tau)=w(t)-H \int_{t-\tau}^{t} w(s) d s-c_{2} \lambda_{i} \Gamma \int_{t-\tau}^{t} w(s-\tau) d s
$$

where $H=J_{1}+c_{1} \lambda_{i} G_{1}$ and $\Gamma=J_{2} /\left(c_{2} \lambda_{i}\right)+G_{2}$. Then systems (5) can be rewritten

$$
\dot{w}(t-\tau)=\left(H+c_{2} \lambda_{i} \Gamma\right) w(t)-c_{2} \lambda_{i} \Gamma H \int_{t-\tau}^{t} w(s) d s-\left(c_{2} \lambda_{i}\right)^{2} \Gamma^{2} \int_{t-\tau}^{t} w(s-\tau) d s
$$


Let $v \in R^{n}$ with components $v_{i}>0(i=1,2, \ldots, n)$ and let us consider the radially unbound Lyapunov functional given by

$$
U(t)=U_{1}(t)+U_{2}(t)+U_{3}(t)+U_{4}(t)
$$

where

$$
\begin{gathered}
U_{1}(t)=\langle|w(t)|, v\rangle \\
U_{2}(t)=\left|c_{2} \lambda_{i}\right|\left\langle|\Gamma H| \int_{-\tau}^{0} \int_{t+s}^{t}|w(\theta)| d \theta d s, v\right\rangle \\
U_{3}(t)=\left(c_{2} \lambda_{i}\right)^{2}\left\langle\left|\Gamma^{2}\right| \int_{-\tau}^{0} \int_{t+s}^{t}|w(\theta-\tau)| d \theta d s, v\right\rangle
\end{gathered}
$$

and

$$
U_{4}(t)=\tau\left(c_{2} \lambda_{i}\right)^{2}\left\langle\left|\Gamma^{2}\right| \int_{t-\tau}^{t}|w(\theta)| d \theta, v\right\rangle
$$

Then it is obvious that

$$
U(t)<\infty, \quad t>0
$$

The right Dini derivative of $U$ along the solution of Equation (8) gives

$$
\left.D^{+} U(t)\right|_{(8)}=\left.D^{+} U_{1}(t)\right|_{(8)}+\left.D^{+} U_{2}(t)\right|_{(8)}+\left.D^{+} U_{3}(t)\right|_{(8)}+\left.D^{+} U_{4}(t)\right|_{(8)}
$$

We have

$$
\left.D^{+} U_{1}(t)\right|_{(8)}=\left\langle\frac{d^{+}|w(t)|}{d t^{+}}, v\right\rangle=\left\langle D_{w}(t) \frac{d^{+} w(t)}{d t^{+}}, v\right\rangle
$$

where $D_{w}(t)=\operatorname{diag}\left\{\operatorname{sgn}\left(w_{1}\right), \operatorname{sgn}\left(w_{2}\right), \ldots, \operatorname{sgn}\left(w_{n}\right)\right\}$. Then we can obtain

$$
\begin{aligned}
\left.D^{+} U_{1}(t)\right|_{(8)} & =\left\langle D_{w}(t)\left(H+c_{2} \lambda_{i} \Gamma\right) w(t), v\right\rangle-\left\langle D_{w}(t)\left(c_{2} \lambda_{i} \Gamma H \int_{t-\tau}^{t} w(s) d s\right), v\right\rangle \\
& -\left\langle D_{w}(t)\left(c_{2} \lambda_{i}\right)^{2} \Gamma^{2} \int_{t-\tau}^{t} w(s-\tau) d s, v\right\rangle
\end{aligned}
$$

Next, by overvaluing $\left.D^{+} U_{1}(t)\right|_{(8)}$, we can get

$$
\begin{aligned}
\left.D^{+} U_{1}(t)\right|_{(8)} \leq & \left\langle\left(H+c_{2} \lambda_{i} \Gamma\right)^{*}|w(t)|, v\right\rangle+\left\langle\left|c_{2} \lambda_{i}\right||\Gamma H| \int_{t-\tau}^{t}|w(s)| d s, v\right\rangle \\
& +\left\langle\left(c_{2} \lambda_{i}\right)^{2}\left|\Gamma^{2}\right| \int_{t-\tau}^{t}|w(s-\tau)| d s, v\right\rangle
\end{aligned}
$$

Similarly, we have

$$
\begin{aligned}
& \left.\left.D^{+} U_{2}(t)\right|_{(8)}=\left|c_{2} \lambda_{i}\right|\langle|\Gamma H|(\tau|w(t)|), v\rangle-\left|c_{2} \lambda_{i}\right|\left\langle|\Gamma H| \int_{t-\tau}^{t}|w(\theta)| d \theta\right), v\right\rangle \\
& \left.D^{+} U_{3}(t)\right|_{(8)}=\left(c_{2} \lambda_{i}\right)^{2}\left\langle\left|\Gamma^{2}\right|(\tau|w(t-\tau)|), v\right\rangle-\left\langle\left(c_{2} \lambda_{i}\right)^{2}\left|\Gamma^{2}\right| \int_{t-\tau}^{t}|w(\theta-\tau)| d \theta, v\right\rangle
\end{aligned}
$$

and

$$
\left.D^{+} U_{4}(t)\right|_{(8)}=\left(c_{2} \lambda_{i}\right)^{2}\left\langle\tau\left|\Gamma^{2}\right|(|w(t)|-|w(t-\tau)|), v\right\rangle
$$

From Eqs. (18)-(21) and Eq. (9), we obtain

$$
\left.D^{+} U(t)\right|_{(8)} \leq\langle-Y|w(t)|, v\rangle
$$

where $A=\left(H+c_{2} \lambda_{i} \Gamma\right)^{*}, B=\left|c_{2} \lambda_{i}\right||\Gamma H|+\left(c_{2} \lambda_{i}\right)^{2}\left|\Gamma^{2}\right|$. 
If $Y$ satisfies the condition (1) and the condition (2), we can find a vector where $\rho \in R_{+}^{*}$ [22], i.e with components $\rho_{k} \in R_{+}^{*}$ satisfying the relation $Y_{1} v=\rho, \forall v \in R_{+}^{*}$, here $\langle-Y|w(t)|, v\rangle=\left\langle-Y_{1} v,|w(t)|\right\rangle$. So, we have

$$
\langle-Y|w(t)|, v\rangle=\langle-\rho,|w(t)|\rangle
$$

In the end, we can get

$$
\left.D^{+} U(t)\right|_{(8)}<-\sum_{k=1}^{n} \rho_{k}\left|w_{k}(t)\right|<0
$$

Then, it follows that zero solution of system (5) is asymptotically stable. Form Lemma 2.1 , we know that the synchronized states (3) are asymptotically stable for the dynamical network (1).The proof is completed.

\section{Numerical Results}

In the previous sections, we discuss a general complex dynamical network.Then a new general stability criterion of synchronization state have been proposed. The above synchronization conditions can be applied to networks with different topologies and different sizes. In order to illustrate the main results of the above theoretical analysis, we consider a lower-dimensional network model with five nodes, in which each node is a simple three-dimensional stable linear system described in the reference [21].

$$
\left\{\begin{array}{l}
\dot{x}_{i 1}(t)=-0.5 x_{i 1}(t)-x_{i 1}(t-\tau) \\
\dot{x}_{i 2}(t)=-x_{i 2}(t)+x_{i 1}(t-\tau)-x_{i 2}(t-\tau) \\
\dot{x}_{i 3}(t)=-1.5 x_{i 3}(t)-x_{i 2}(t-\tau)
\end{array}\right.
$$

which is asymptotically stable at $s(t)=0$, and Jacobian matrices are $J_{I}(t)=\operatorname{diag}\{-0.5,-1$, $-1.5\}$ and $J_{2}(t)=\operatorname{diag}\{-1,0,0 ; 1,-1,0 ; 0,0,-1\}$.

Assume that the outer-coupling matrix $C$ and the inner-coupling matrix $G_{1}, G_{2}$ are given as follows

$$
C=\left[\begin{array}{ccccc}
-2 & 1 & 0 & 0 & 1 \\
1 & -3 & 1 & 1 & 0 \\
0 & 1 & -2 & 1 & 0 \\
0 & 1 & 1 & -3 & 1 \\
1 & 0 & 0 & 1 & -2
\end{array}\right] \quad G_{1}=\left[\begin{array}{cccc}
1.8090 & 0 & 0 \\
0 & 3.6179 & 0 \\
0 & 0 & 5.4269
\end{array}\right] \quad G_{2}=\left[\begin{array}{ccc}
-2.0912 & 1.2382 & 0 \\
1.7355 & -3.6179 & 1.0337 \\
0 & 1.4472 & -3.6179
\end{array}\right]
$$

Obviously, $C$ is an irreducible symmetric matrix. The eigenvalues of $C$ are $\lambda_{i}=0$, $1.382,-2.382,-3.618,-4.618$. For clearer visions, we take the coupling strength $c_{1}=c_{2}=0.1$ and $\tau=0.05$.

In terms of Theorem 3.1, if the condition (1) and the condition (2) are satisfied, then it is inferred that the synchronization of the complex network (1) can be achieved. When $\lambda_{i}$ $=-1.382,-2.382,-3.618,-4.618$, we can get $H=\operatorname{diag}\{-0.7500,-1.5000,-2.2500\}, H=\operatorname{diag}\{-$ $0.9309,-1.8618,-2.7927\}, H=\operatorname{diag}\{-1.1545,-2.3090,-3.4635\}, H=\operatorname{diag}\{-1.3354,-2.6707$, $-4.0061\}$, and

$$
\Gamma=\left[\begin{array}{ccc}
5.1447 & 1.2382 & 0 \\
-5.5004 & 3.6180 & 1.0337 \\
0 & 1.4472 & 3.6180
\end{array}\right], \quad \Gamma=\left[\begin{array}{ccc}
2.1070 & 1.2382 & 0 \\
-2.4627 & 0.5803 & 1.0337 \\
0 & 1.4472 & 0.5803
\end{array}\right],
$$




$$
\Gamma=\left[\begin{array}{ccc}
0.6728 & 1.2382 & 0 \\
-1.0285 & -0.8539 & 1.0337 \\
0 & 1.4472 & -0.8539
\end{array}\right], \quad \Gamma=\left[\begin{array}{ccc}
0.0742 & 1.2382 & 0 \\
-0.4299 & -1.4525 & 1.0337 \\
0 & 1.4472 & -1.4525
\end{array}\right]
$$

, respectively. Then we easily obtain

$$
\begin{aligned}
& Y=\left[\begin{array}{ccc}
1.6567 & -0.1986 & -0.0012 \\
-0.8442 & 2.4426 & -0.1721 \\
-0.0076 & -0.2300 & 3.4111
\end{array}\right], \quad Y=\left[\begin{array}{ccc}
1.4728 & -0.3339 & -0.0036 \\
-0.6437 & 2.1210 & -0.2877 \\
-0.0101 & -0.3840 & 3.1123
\end{array}\right] \text {, } \\
& Y=\left[\begin{array}{ccc}
1.2259 & -0.4942 & -0.0084 \\
-0.3913 & 1.6539 & -0.4434 \\
-0.0097 & -0.5921 & 2.6301
\end{array}\right] \text { and } Y=\left[\begin{array}{ccc}
1.0270 & -0.6472 & -0.0137 \\
-0.2148 & 1.2293 & -0.5832 \\
-0.0066 & -0.7800 & 2.1901
\end{array}\right] \text {, }
\end{aligned}
$$
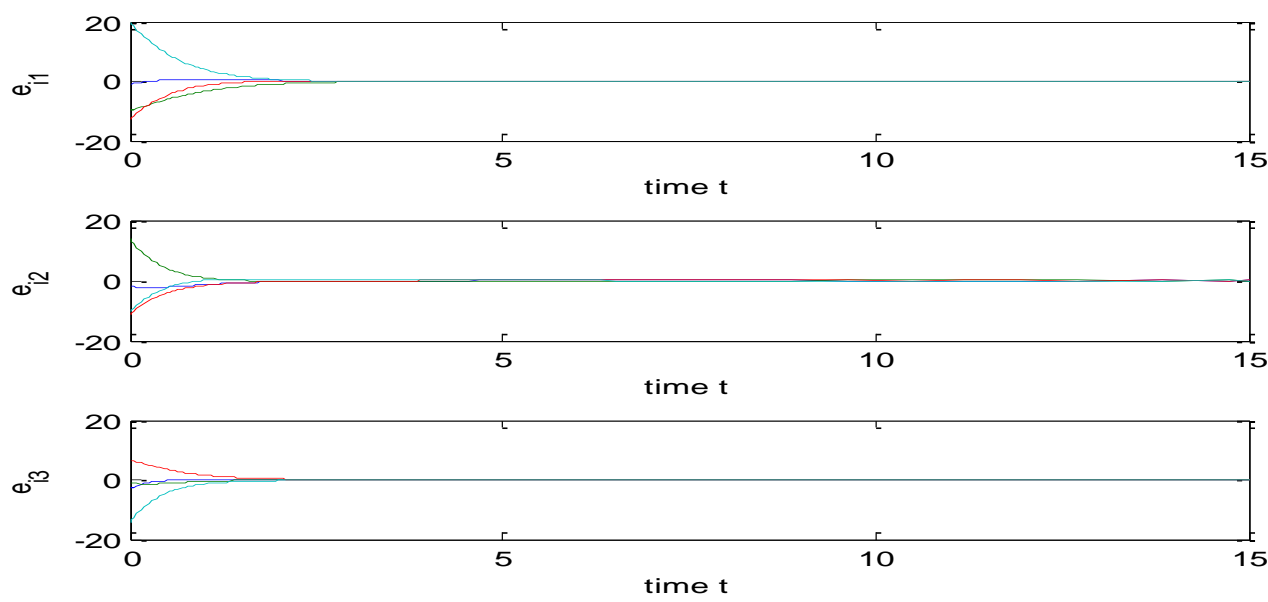

Figure 1 (Color Online). Synchronization Errors for the Delayed Network with Coupling Strength $c 1=0.1, c 2=0.1$ and Time Delay $\tau=0.05$
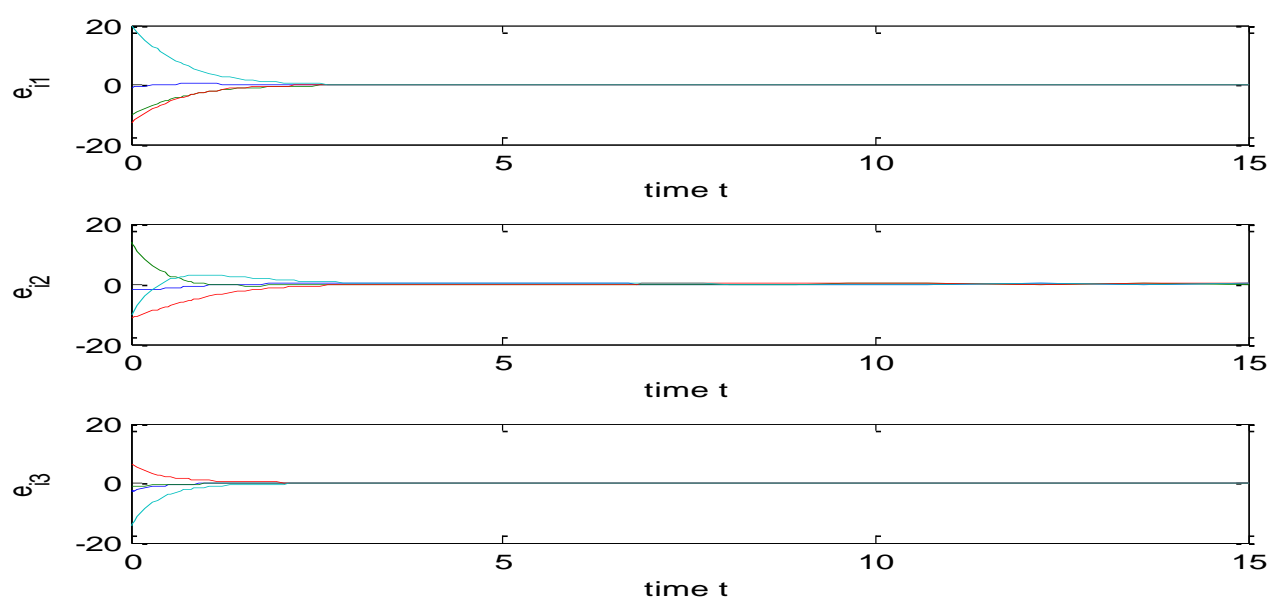

Figure 2 (Color Online). Synchronization Errors for the Delayed network with Coupling Strength $c 1=0.01, c 2=0.03$ and Time Delay $\tau=0.1$ 
respectively. Obviously, the condition (1) of Theorem 3.1 is satisfied. At the same time, we observe that eigenvalues of the above four matrices are $d_{1}=(1.4787,2.5777,3.4539)$, $d_{2}=(1.2201,2.2622,3.2237), d_{3}=(0.9901,1.7422,2.8675)$ and $d_{4}=(0.6053,1.2853$, 2.5558), respectively. Therefore, the condition (2) of Theorem 3.1 is satisfied and the synchronized states (3) of network (1) are asymptotically stable. In Figure 1, we plot the curves of the synchronization errors between the states of node $i$ and node $i+l$ (that is, $\left.e_{i j}(t)=x_{i j}(t)-x_{i+1, j}(t)\right)$, for $i=1,2,3,4, j=1,2,3$, with the coupling strength $c_{1}=c_{2}=0.1$ and time delay $\tau=0.05$. When the coupling strength $c_{1}=0.01, c_{2}=0.03$ and time delay $\tau=0.1$ and the other parameters are the same as the above example, we can also derive the conclusion that the two conditions of Theorem 3.1 are satisfied. The curves of the synchronization errors are shown in Figure 2. We see that the synchronization errors converge to zero under the above conditions.

\section{Conclusions}

In the paper, we introduce a general complex dynamical network model with nondelayed and delayed coupling. According to the stability theory of the linear time-delay system, we have obtained a new general stability criterion of synchronization state in the complex dynamical networks. Making full use of constructing the Lyapunov function, the criterion is easy to be verified. In the end, some numerical simulations are in line with the theoretical analyses.

\section{Acknowledgements}

This work was supported by the Natural Science Foundation of China under Grant No. 61231002, No. 51075068 and No. 61573004, by Cloud Computing Platform for Internet of Things-Fujian Scientific Research Platform for Innovation under Grant No. 2013H2002, by the Foundation of the Fujian Education Department under Grant No. JA15035, by the Foundation of Quanzhou under Grant No. 2014Z103, No. $2015 Z 114$ and No. 2014Z113. The authors would like to thank the reviewers for their valuable suggestions and comments.

\section{References}

[1] D. J. Watts and S. H. Strogatz, "Collective Dynamics of Small-world Networks", Nature, vol. 393, no. 1, (1998), pp.440-442.

[2] A. -L. Barabási and R. Albert, "Emergence of Scaling in Random Networks", Science, vol. 286, no. 5439, (1999), pp.509-512.

[3] R. Albert and A. -L. Barabási, "Statistical Mechanics of Complex Networks", Rev. Modern Phys., vol. 74, no. 1, (2002), pp. 47-97.

[4] X. F. Wang and G. R. Chen, "Synchronization in Scale-free Dynamical Networks: Robustness and Fragility”, IEEE Trans. Circuits Syst. I, vol. 49, no. 1, (2002), pp. 54-62.

[5] J. N. Tang and P. Z. Liu, "Synchronization in a Novel Local-World Dynamical Network Model", Math. Probl. Eng.,vol. 2014, (2014), pp. 851403.

[6] J. N. Tang, C. R. Zou, L. Zhao, X. Z. Xu and X. M. Du, "Impulsive Stabilization for Control and Synchronization of Complex Networks with Coupling Delays”, J. Phys. Soc. Jpn., vol. 81, no.1, (2012), pp. 014003.

[7] J. N. Tang, C. R. Zou, S. P. Wang, L. Zhao and X. P. Liu, "Chaos Synchronization of Chen Systems with Time-varying Delays", Int. J. Bifurcation Chaos, vol. 22, no.8, (2012), pp.1250183.

[8] J. Lu, J. Kurths, J. Cao, N. Mahdavi and C. Huang, "Synchronization Control for Nonlinear Stochastic Dynamical Networks: Pinning Impulsive Strategy", IEEE Trans. Neural Netw. Learn. Syst., vol. 23, no. 2, (2012), pp. 285-292.

[9] J. N. Tang, C. R. Zou and L. Zhao, "A General Complex Dynamical Network With Time-Varying Delays and Its Novel Controlled Synchronization Criteria”, IEEE Syst. J., vol. 99, (2014), pp.1-7.

[10] Z. G. Wu, P. Shi, H. Y. Su and J. Chu, "Sampled-Data Exponential Synchronization of Complex Dynamical Networks With Time-Varying Coupling Delay", IEEE Trans. Neural Netw. Learn. Syst., vol. 24, no. 8, (2013), pp. 1177-1187.

[11 ]C. G. Li and G. R. Chen, "Synchronization in General Complex Dynamical Networks with Coupling Delays", Physica A, vol. 343, (2004), pp.263-278. 
[12] J. H. Lǔ and G. Chen, "A Time-varying Complex Dynamical Network Model and Its Controlled Synchronization Criteria”, IEEE Trans. Automat. Contr., vol. 50, no. 6, (2005), pp.841-846.

[13] H. J. Gao, J. Lam and G. R. Chen, "New Criteria for Synchronization Stability of General Complex Dynamical Networks with Coupling Delays", Phys. Lett. A, vol. 360, no. 2, (2006), pp. 263-273.

[14] J. S. Wu, L. C. Jiao, "Synchronization in Complex Delayed Dynamical Networks with Nonsymmetric Coupling”, Physica A, vol. 386, no. 1, (2007), pp. 513-530.

[15] K. Li, S. G. Guan, X. F. Gong and C. H. Lai, "Synchronization Stability of General Complex Dynamical Networks with Time-varying Delays", Phys. Lett. A, vol. 372, no. 48, (2008), pp. 7133-7139.

[16] H. G. Zhang, D. W. Gong and Z. S. Wang, "New Synchronization Analysis for Complex Networks with Variable Delays", Chinese Phys. B, vol. 20, no. 4, (2011), pp. 040512.

[17] N. Li, Y. L. Zhang, J. W. Hu and Z. Y. Nie, "Synchronization for General Complex Dynamical Networks with Sampled-data", Neurocomputing, vol. 74, no.5, (2011), pp. 805-811.

[18] W. Lu, T. Chen and G. Chen, "Synchronization Analysis of Linearly Coupled Systems Described by Differential Equations with a Coupling Delay", Physica D, vol. 221, no. 2, (2006), pp. 118-134.

[19] C. Hu, J. Yu, H. Jiang and Z. Teng, "Exponential Synchronization of Complex Networks With Finite Distributed Delays Coupling”, IEEE T. Neural Networ., vol. 22, no. 12, (2011), pp. 1999-2010.

[20] Y. Liang, X. Y. Wang and J. Eustace, "Adaptive Synchronization in Complex Networks with Non-delay and Variable Delay Couplings via Pinning Control", Neurocomputing, vol. 123, (2014), pp. 292-298.

[21] H. J. Li, "New Criteria for Synchronization Stability of Continuous Complex Dynamical Networks with Non-delayed and Delayed Coupling”, Commun. Nonlinear. Sci. Numer. Simular., vol. 16, no. 2, (2011), pp.1027-1043.

[22] J. C. Gentina, P. Bome and F. Laurent, "Stabilitédes Sytèmes Continus non Linéaires de Grande Dimension", RAIRO Revue jaune, vol. J3, (1972), pp. 69-72.

\section{Authors}
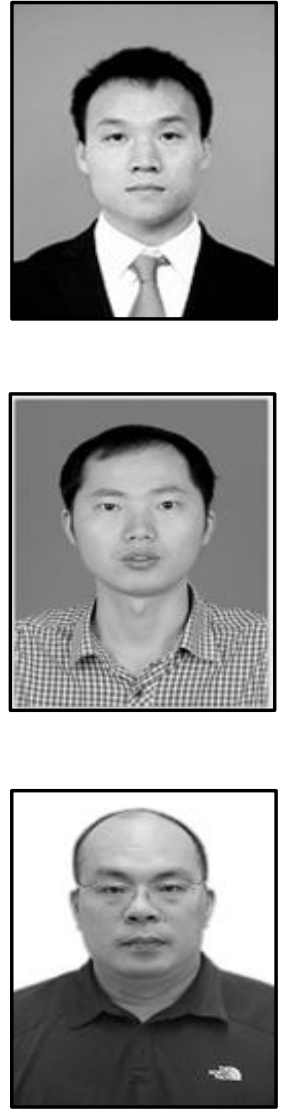

Peizhong Liu, he was born in 1976. He received the P. H. degree from school of information science and engineering, Xiamen University, Xiamen, Fujian, China. Now he is an IOT Experimentalist, and his research interests include multi-dimensional space biomimetic informatics, visual media retrieval, network model, information security. degree in Electric Circuit and System from University of Chinese Academy of Sciences in 2013. Currently, he is with College of Engineering, Huaqiao University, Quanzhou, Fujian, China. His main research interests include chaos synchronization, nonlinear systems, complex networks, digital image processing and target tracking.

Jianeng Tang, he was born in 1983. He received the $\mathrm{Ph}$. D. degree in Information and Communication Engineering from Southeast University in 2012. Currently, he is with College of Engineering, Huaqiao University, Quanzhou, Fujian, China. His main research interests include nonlinear systems, chaos synchronization and control, complex networks, acoustic emission technology and speech signal processing.

$$
\text { complex networks, digital image processing and target tracking. }
$$

Detian Huang, he was born in 1985 . He received the $\mathrm{Ph}$. D. 


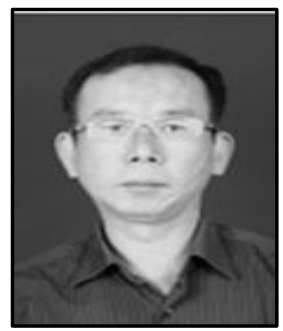

Yuzhao Zhang, he was born in 1963. He received the B.S. degree in Electronic Instrumentation and Measurement Technology from Southeast University in 1983. Currently, he is with College of Engineering, Huaqiao University, Quanzhou, Fujian, China. His research interests include chaos synchronization and control, complex networks, speech signal processing and wireless communication. 
International Journal of Control and Automation Vol. 9, No. 9 (2016) 